\title{
SKLB-163, a new benzothiazole-2-thiol derivative, exhibits potent anticancer activity by affecting RhoGDI/JNK-1 signaling pathway
}

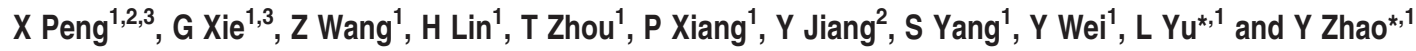

Small-molecule inhibitors are an attractive therapeutic approach for most types of human cancers. SKLB-163, a novel benzothiazole-2-thiol derivative, was developed via computer-aided drug design and de novo synthesis. MTT assay showed it had potent anti-proliferative activity on various human cancer cells. Treatment of cancer cells with SKLB-163 induced obvious apoptosis and inhibited proliferation in vitro. SKLB-163 administered p.o. showed a marked antitumor activity in vivo. Proteomic techniques were employed to identify possible drug target proteins. The data showed molecular mechanism of action might be involved in downregulation of RhoGDI, which finally contributed to increased apoptosis and inhibited proliferation. These findings provided the potential value of SKLB-163 as a novel candidate antitumor drug.

Cell Death and Disease (2014) 5, e1143; doi:10.1038/cddis.2014.107; published online 27 March 2014

Subject Category: Cancer

The cancer is notably complex, widespread and lethal disease, which can affect almost every tissue lineage in our bodies and arise from normal cells as a result of diverse gene mutations. ${ }^{1}$ Surgery, chemotherapy and radiotherapy are the main choices of current cancer treatment. Although advances in the therapy of cancer have been made over the past decade, it continues to pose great challenges to medical science. ${ }^{2}$ Thus, it is an urgent need to discover and develop novel antitumor drugs. Small-molecule inhibitors that target specific signal pathways in tumor cells are a very promising class of drugs for the treatment of cancers, such as imatinib and gefitinib. 3,4

Benzothiazole derivatives have been known for different biological properties, including antimalarial, antitubercular, antihelmintic, anticonvulsant, analgesic, anti-inflammatory, antidiabetic and antitumor activities. ${ }^{5,6}$ It has been reported that the benzothiazole moiety modified with some functional groups, such as aryl and imidazole, can significantly inhibit the growth of certain cancer cell lines. ${ }^{7,8,9}$ However, most studies focused on designing new benzothiazole compounds by substituting 2-aminobenzothiazoles or 2-arylbenzothiazoles; only a few researchers employed the benzothiazole-2-thiol as a functional group. Recently, a novel series of benzothiazole2-thiol derivatives were synthesized in our laboratory and most of the compounds exhibited good inhibitory activities on cancer cell growth in vitro. Moreover, some of them were more effective than cisplatin. ${ }^{10}$ Among these compounds, SKLB163 exhibited very good antitumor activities against many different types of human cancer cell lines with $\mathrm{IC}_{50}$ values in the low micromolar range. However, the exact biological effects of SKLB-163 have not been reported. In this paper, we present the results of the evaluation of the in vivo antitumor activity and toxicity of SKLB-163 against several human tumors xenografted in nude mice and attempt to study its possible action mechanism.

\section{Results}

The cytotoxicity effect of SKLB-163 on tumor cells. It has been reported that SKLB-163 was synthesized in our laboratory (Figure 1) and exhibited good inhibitory activities on many types of cancer cell in vitro. Moreover, five cancer cell lines (A375, SPC-A1, SW620, Hela and PC-3) were used to investigate the cytotoxicity of SKLB-163 in this study. After $48 \mathrm{~h}$ of treatment, SKLB-163 inhibited viability of all cell lines. The IC50 for A375, SPC-A1, SW620, Hela and PC-3 were shown in Table 1. In the light of data from previous and our study, A375 and SPC-A1 cells were more sensitive to SKLB163 treatment than others and thus were chosen for further study.

Induction of apoptosis and suppression of proliferation by SKLB-163. Fluorescence microscopic examination of Hoechst-stained cell was performed to assess the apoptosis induction effect of SKLB-163 in A375 cells. Treatment with SKLB-163 resulted in morphological changes characteristic for apoptosis: a brightly blue-fluorescent condensed nuclei (intact or fragmented), condensation of nuclear chromatin,

\footnotetext{
${ }^{1}$ State Key Laboratory of Biotherapy and Cancer Center, West China Hospital, West China Medical School, Sichuan University, Chengdu, Sichuan, China and ${ }^{2}$ Department of Medical Oncology, Cancer Center, State Key Laboratory of Biotherapy, West China Hospital, Sichuan University, Chengdu, Sichuan, China ${ }^{*}$ Corresponding author: L Yu or Y Zhao, State Key Laboratory of Biotherapy and Cancer Center, West China Hospital, West China Medical School, Sichuan University, Chengdu, Sichuan, China. Tel: + 8628 85164059; Fax: + 8628 85164060; E-mail: yuluot@scu.edu.cn or alancenxb@sina.com.cn

${ }^{3}$ These authors contributed equally to this work.

Keywords: benzothiazole-2-thiol derivative; cancer; RhoGDI; apoptosis; proliferation

Abbreviations: SKLB-163, a novel benzothiazole-2-thiol derivative; TUNEL, terminal deoxynucleotidyl transferase-mediated dUTP nick-end labeling; 2-DE, Two-dimensional gel electrophoresis; ESI-Q-TOF, electrospray ionization quadrupole time-of-flight; RhoGDI, Rho GDP-dissociation inhibitor 1

Received 05.12.13; revised 21.1.14; accepted 21.1.14; Edited by A Stephanou
} 


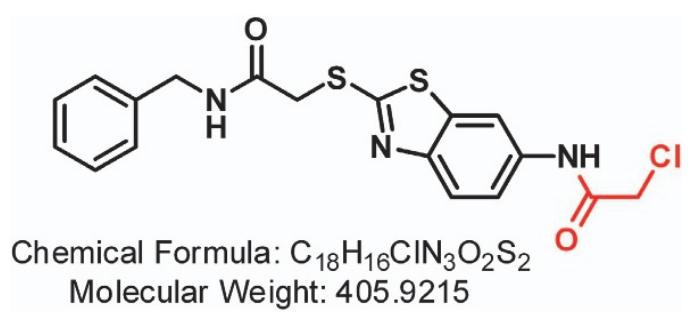

Figure 1 The structure of SKLB-163

Table 1 The cytotoxicity effect of SKLB-163 on tumor cells

\begin{tabular}{llc}
\hline $\begin{array}{l}\text { Tumor } \\
\text { cell line }\end{array}$ & Cell type & $\begin{array}{c}\text { IC50 }(\boldsymbol{\mu} \text { M) } \\
(\text { mean } \pm \text { S.D.) }\end{array}$ \\
\hline A375 & Human melanoma cell line & $1.82 \pm 0.25$ \\
SPC-A1 & Human lung adenocarcinoma cell line & $3.89 \pm 0.64$ \\
SW620 & Human colorectal cancer cell line & $5.12 \pm 0.98$ \\
Hela & Human cervical cancer cell line & $5.13 \pm 1.09$ \\
PC-3 & Human prostate cancer cell line & $6.58 \pm 1.05$ \\
\hline
\end{tabular}

Each cell line was treated with various concentrations $(0-20 \mu \mathrm{M})$ of SKLB-163 for $48 \mathrm{~h}$, respectively. Cell viability was detected by MTT assay. Data are expressed as the mean \pm S.D. from three experiments

nuclear fragmentation, and apoptotic bodies, and the change was concentration-dependent (Figure 2A).

In addition, the quantitative assessment of A375 cells with sub-G1 DNA content by flow cytometry was used to estimate the number of apoptotic cells. As shown in Figure 2B, there was a marked increase in the sub-G1 peak from $5.8 \%$ in the untreated cells to $16.6 \%, 29.1 \%$, and $54 \%$, respectively, in $0.625,1.25$, and $2.5 \mu \mathrm{M}$ SKLB-163-treated cells for $48 \mathrm{~h}$. These results suggested that SKLB-163 could induce apoptosis in A375 cells in a dose-dependent manner in vitro.

Furthermore, SKLB-163 resulted in remarkable inhibition of tumor cell proliferation that was demonstrated by clonogenic formation assays. Clonogenic formation assays demonstrated that upon 14-day continuous culture, there was a marked inhibition in the clone number from $324 \pm 32.9$ in the untreated $A 375$ cells to $231 \pm 26.1,154 \pm 25.3$, and $72 \pm 36.3$, respectively, in $0.625,1.25$, and $2.5 \mu \mathrm{M}$ SKLB-163-treated cells (Dunnett's t-test, $P<0.05$ ) (Figure 2C). These data showed that the potential to form colonies of A375 cells was inhibited by SKLB-163 in a dose-dependent form.

SKLB-163 significantly inhibited tumor growth in vivo. The in vivo effects were explored in $A 375$ and SPC-A1 tumor models. As shown in Figure 3, treatments with SKLB-163 at the doses of 100 and $200 \mathrm{mg} / \mathrm{kg}$ significantly reduced the rates of primary tumor growth in comparison with control $(P<0.05$, respectively) as evaluated by measuring tumor volume at regular intervals. The similar results were also found in the tumor weight. All the data showed that SKLB-163 has a significant influence on the suppression of tumor growth.

SKLB-163 decreased cell proliferation and increased apoptosis in vivo. It was apparent that SKLB-163 induced apoptosis and inhibited cell proliferation in vitro. To obtain additional insight into the in vivo effects, tumor cell proliferation and apoptosis were assessed by PCNA immunoreactivity analysis and TUNEL assay. As shown in Figure 4, SKLB-163 clearly reduced percentages of PCNA-positive nuclei in A375 tumor models. Moreover, in the TUNEL assay to evaluate apoptosis in vivo, a significantly greater percentage of TUNEL-positive nuclei could be observed in A375 tumors treated with SKLB-163 in a concentration-dependent way when compared with the tumors from control group. Thus, SKLB-163 could significantly inhibit cell proliferation and induce apoptosis in vivo.

Toxicity assessment. To evaluate the possible adverse effects of SKLB-163, weight of mice was monitored every 3 days throughout the whole experiment. The weight curve of SKLB-163-treated group (including 50, 100, and $200 \mathrm{mg} / \mathrm{kg}$ ) paralleled very closely that of the control group (Figure 5a). No ruffled fur or toxic death was observed in the SKLB-163treated group.

The levels of serum AST and ALT of the mice treated with SKLB-163 were determined to evaluate the liver toxicity. No significant elevation was observed in the SKLB-163-treated group compared with the control group $(P>0.05)$ (Figure $5 b)$.

Furthermore, no toxic pathological changes in heart, liver, lung, spleen, kidney were found via microscopic examination (Figure 5c). White blood cell, platelet, red blood cell counts, and hemoglobin concentration of the mice were determined to assess the potential blood toxicity induced by SKLB-163 treatment. No significant changes were observed in the SKLB-163-treated group compared with the control group $(P>0.05$, respectively) (Table 2$)$.

2-DE profiling of the differentially expressed proteins between A375 and SKLB-163-treated A375 cells. The protein expression profile of A375 cells and SKLB-163treated A375 cells were examined by two-dimensional electrophoresis. The experiments were repeated three times. The image analysis was conducted by using PDQuest 6.1 software and showed that these 2-DE maps were reproducible (Figure 6a). Differentially expressed proteins were defined as statistically significant based on two criteria: 1) intensity alterations $>$ twofold (Student's $t$-test, $P<0.05$ ) and 2) recurrence more than two times in the three repeated experiments. According to these criteria, a total of 21 unique proteins were identified by ESI-Q-TOF-MS/MS, as listed in Table 3. Cluster analysis revealed that the altered proteins were involved in diverse biological processes, including metabolism (19\%), transcription regulation (14.3\%), proteolysis $(14.3 \%)$ and so on (Figure 6b). The identified proteins were categorized into groups according to their subcellular locations. In all, $57 \%$ of the proteins were located in the cytoplasm and others were located in the nucleus $(29 \%)$ or membrane $(14 \%)$ as indicated (Figure 6c). Among them, Rho GDP-dissociation inhibitor 1 (RhoGDI) was identified with very significant alteration. It was downregulated 3.6-fold in SKLB-163-treated A375 cells compared with A375 cells $(P<0.05)$. Further, ESI-Q-TOF-MS/MS analysis revealed that RhoGDI has 12 matched peptides and a MOWSE score of 135.

SKLB-163 downregulated RhoGDI. RhoGDI was further validated by western blot. Consistent with the observations in 

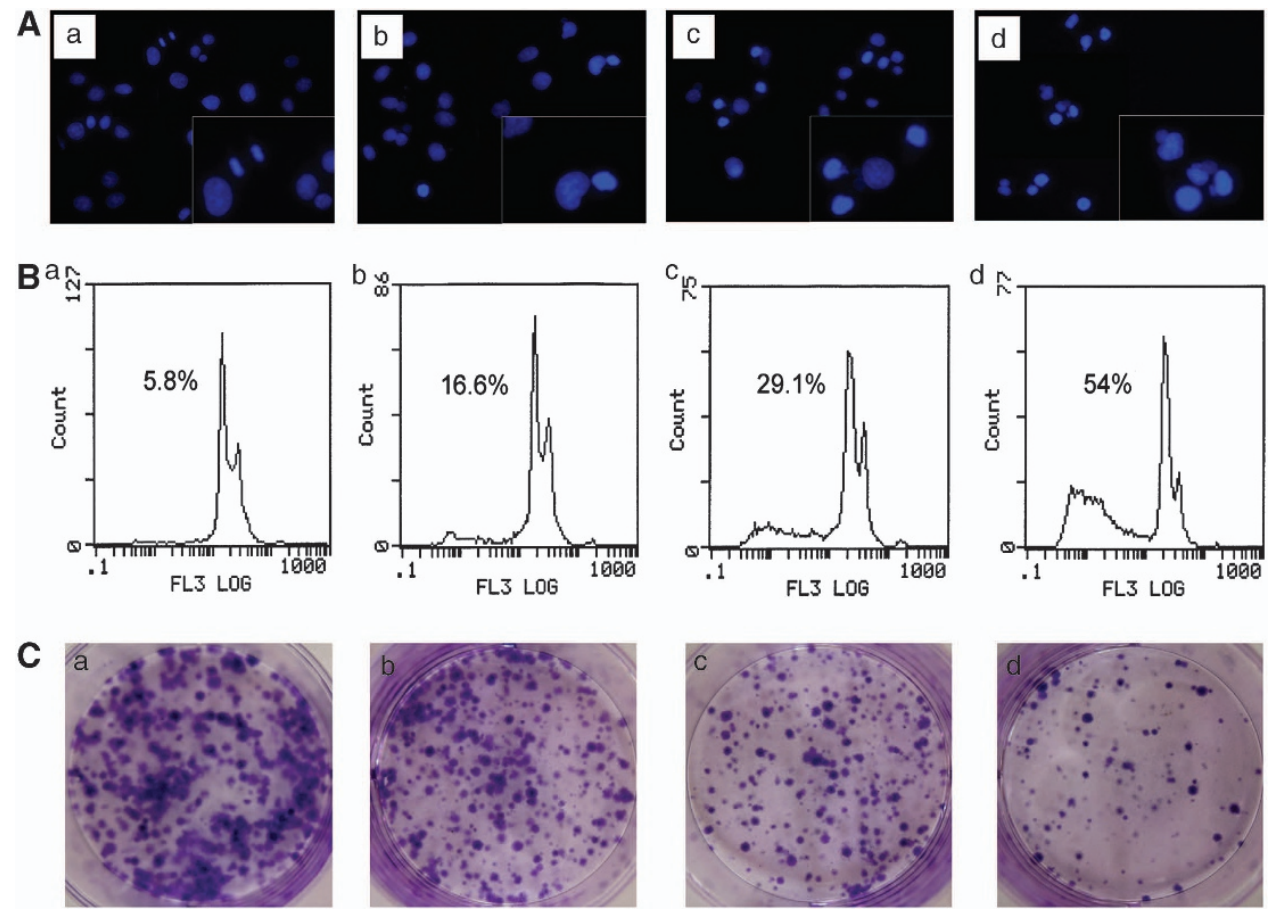

Figure 2 SKLB-163 could induce apoptosis and inhibit proliferation in vitro. (A) The fluorescence microscopic appearance of Hoechst-stained nuclei of A375 cells after incubation with SKLB-163 for $48 \mathrm{~h}$ at varying concentrations: $0 \mu \mathrm{M}(\mathrm{a}) ; 0.625 \mu \mathrm{M}(\mathrm{b}) ; 1.25 \mu \mathrm{M}(\mathrm{c}) ; 2.5 \mu \mathrm{M}$ (d). Apoptotic cells containing condensed and fragmented fluorescent nuclei were visible in SKLB-163-treated cells, whereas the untreated cells showed blue, diffusely stained intact nuclei. (B) Representative DNA fluorescence histograms of PI-stained cells. After incubated with $0 \mu \mathrm{M}(\mathrm{a}) ; 0.625 \mu \mathrm{M}(\mathrm{b}) ; 1.25 \mu \mathrm{M}(\mathrm{c}) ; 2.5 \mu \mathrm{M}(\mathrm{d})$ of SKLB-163 for $48 \mathrm{~h}$, there was a marked increase in the sub-G1 peak in a dose-dependent way. (C) Results of colony-forming assays showed that upon 14-day continuous culture, there was a marked inhibition in the clone number from $324 \pm 32.9$ in the untreated A375 cells (a) to $231 \pm 26.1,154 \pm 25.3,72 \pm 36.3$, respectively, in 0.625 (b), 1.25 (c) and $2.5 \mu \mathrm{M}$ (d) SKLB-163-treated A375 cells

2-DE analysis, RhoGDI was downregulated particularly in the SKLB-163-treated A375 cells compared with the parental A375 cells (Figure 7a). A similar phenomenon was also observed in the SKLB-163-treated SPC-A1 cells (data not shown).

In addition, we attempted to overexpress RhoGDI to check whether it would affect antitumor effect of SKLB-163. As shown in Figures $7 \mathrm{~b}$ and $\mathrm{c}$, overexpression of RhoGDI markedly rescued tumor cells from SKLB-163-induced tumor suppression in vitro and in vivo, which suggested target specificity.

SKLB-163 activated JNK-1 signaling pathway. It has been reported that RhoGDI inhibition could activate JNK signaling pathway, which is involved in the regulation of apoptosis and proliferation. ${ }^{11}$ To further investigate the molecular mechanism of SKLB-163, we used western blotting to examine the phosphorylation activities in the JNK signaling pathway. As shown in Figure $7 d$, the phosphorylation level of JNK was upregulated dosedependently in tumor cells treated with SKLB-163. Moreover, the phosphorylation level of JNK downstream target c-jun was also elevated accordingly.

JNK isoforms have distinct function in cancer. Thus, we assessed the effects of JNK-1 or JNK-2 silencing on antitumor effect of SKLB-163. Our results showed silencing JNK-1, but not JNK-2, could block SKLB-163 mediated tumor suppression in vitro and in vivo (Figures $7 \mathrm{e}-\mathrm{g}$ ). These results above showed that SKLB-163 inhibited RhoGDI and activated JNK-1 signaling pathway.

Activated caspase-3 and reduced phosphorylated Akt and p44/42 MAPK by SKLB-163. Caspase-3 is an effecter caspase that has a central role in cell apoptosis. Therefore, we investigated the effect of SKLB-163 on the activation of caspase-3. Treatment of A375 cells with SKLB-163 for $48 \mathrm{~h}$ resulted in a concentration-dependent increase of cleavage caspase-3 (Figure 7h).

Furthermore, we studied whether Akt and p44/42 MAPK, which are known to be very important in cell growth and survival, were involved in SKLB-163-mediated proliferation inhibitory effect. Our result showed SKLB-163 clearly reduced phosphorylated Akt and phosphorylated p44/42 MAPK in a concentration-dependent way (Figure 7h), indicating that SKLB-163 could alter signaling to Akt and MAPK. The levels of total Akt and MAPK were not visibly changed, however, the phosphorylated form, as the main part of this protein system, was inhibited much more significantly.

Finally, in order to better illustrate the action mechanism of SKLB-163, a schematic model was proposed based on published literature and the present study (Figure 7i). Taken together, SKLB-163 inhibited upstream RhoGDI and activated JNK-1 signaling pathway that could affect cellular proliferation and apoptosis. 

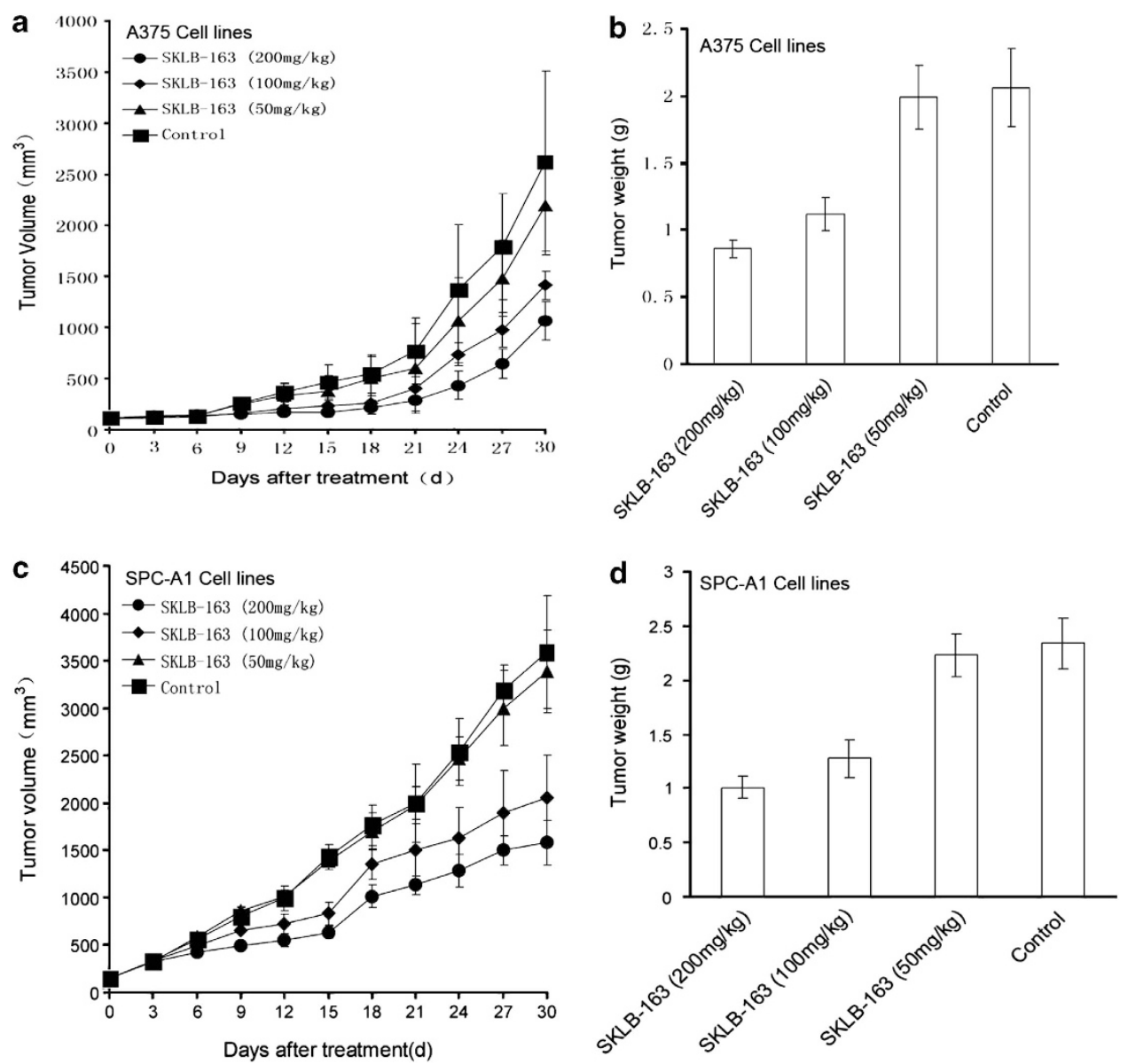

Figure 3 Antitumor effects of SKLB-163 in female athymic nude BALB/c mice bearing human melanoma $A 375$ or lung adenocarcinoma SPC-A1. $4 \times 10^{6} \mathrm{~A} 375$ or $5 \times 10^{6}$ SPC-A1 cells were subcutaneously injected into the right dorsal flank of 6- to 8-week-old nude BALB/c mice. When the tumor diameters were about $0.6-0.8 \mathrm{~cm}$, the tumorbearing mice were randomly assigned into the following four groups and each mouse received the corresponding treatment by intragastric administration once daily: control group, $0.5 \%$ carboxymethylcellulose; SKLB-163, $50 \mathrm{mg} / \mathrm{kg}$; SKLB-163, $100 \mathrm{mg} / \mathrm{kg}$; SKLB-163, $200 \mathrm{mg} / \mathrm{kg}$. Tumor size was measured at the indicated days and volume was calculated as described in 'Materials and Methods.' Effects of SKLB-163 on the growth curves of A375 (a) and SPC-A1 (c) and effects on the tumor weight changes of animals in the A375 (b) and SPC-A1 models (d). Tumor growth was significantly inhibited in the SKLB-163-treated group compared with the control (especially at the doses of 100 and $200 \mathrm{mg} / \mathrm{kg}$ ). The similar results were also found in the tumor weight. Each group experiment was done twice

\section{Discussion}

Molecular targeted therapies, based on recent advances in cancer molecular biology and genomics, have shown promise in the management of various malignancies, with lower toxicity profiles and better overall survival as compared with conventional therapy. ${ }^{12,13}$

In this study, we investigated the biological activities of SKLB-163, a new benzothiazole-2-thiol derivative, in detail. It was developed via computer-aided drug design and de novo synthesis. SKLB-163 showed significant cytotoxicity against various human cancer cells by MTT assay. Obvious suppression of tumor cell proliferation and induction of apoptosis were evidenced by Hoechst staining, flow cytometry and colony formation assay. The in vivo effects were explored in A375 and SPC-A1 tumor models. SKLB-163 administered p.o. displayed a marked antitumor activity. To obtain additional insight into the in vivo effects, tumor cell proliferation and apoptosis were assessed by PCNA immunoreactivity analysis and TUNEL assay. SKLB-163 clearly reduced percentages of
PCNA-positive nuclei and increased percentages of TUNELpositive nuclei in a concentration-dependent way. Potential toxicity induced by SKLB-163 treatment was not observed throughout the whole experiment. 2-DE and ESI-Q-TOF-MS/MS were utilized to identify possible drug target proteins. RhoGDI was downregulated 3.6-fold in SKLB-163-treated tumor cells compared with the control and has 12 matched peptides and a MOWSE score of 135 , which was further validated by western blot. In addition, overexpression of RhoGDI markedly rescued tumor cells from SKLB-163induced tumor suppression, which suggested target specificity. To further investigate the molecular mechanism of SKLB-163, we used western blot to examine the phosphorylation activities in the JNK signaling pathway, which is downstream pathway of RhoGDI and can control apoptosis and proliferation of cancer cells. The phosphorylation level of JNK was upregulated dose-dependently in tumor cells treated with SKLB-163. Moreover, the phosphorylation level of JNK downstream target c-jun was also elevated accordingly. In addition, we found silencing JNK-1, but not JNK-2, could 
a
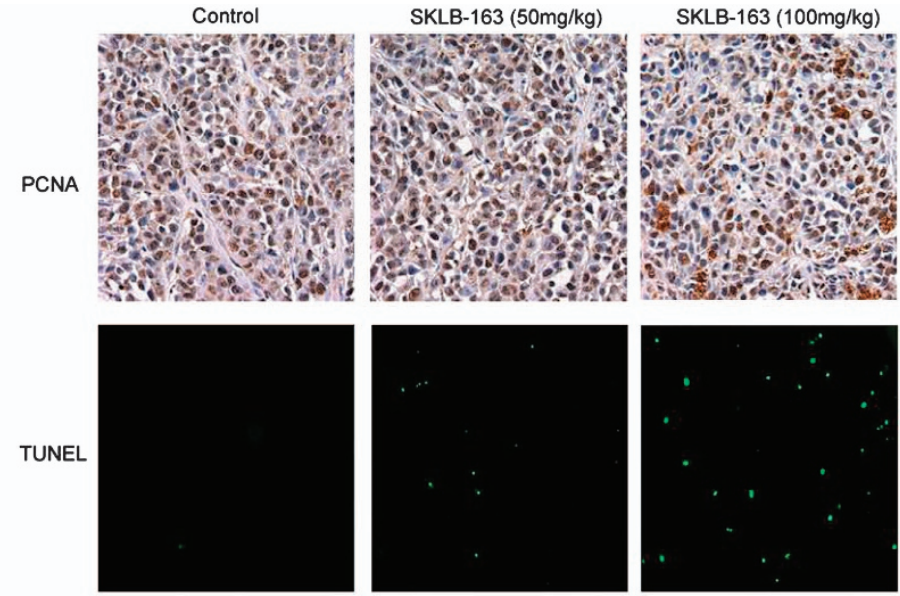

SKLB-163 $(200 \mathrm{mg} / \mathrm{kg})$
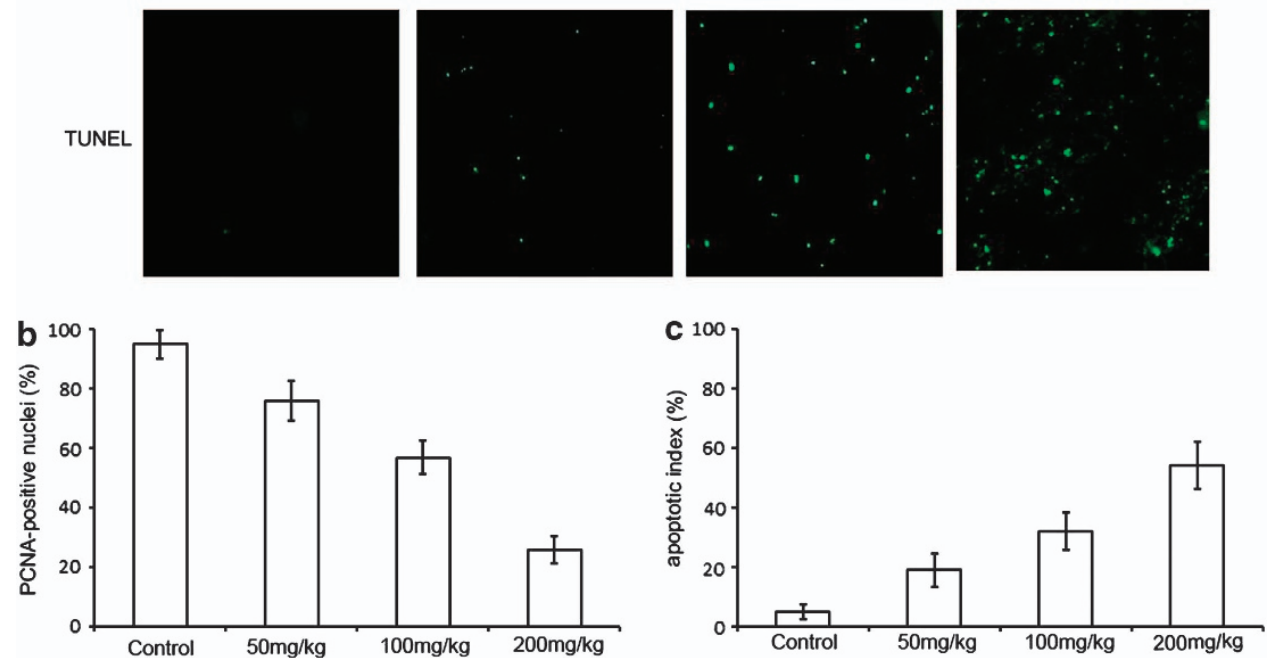

Figure 4 Histological analysis by PCNA immunoreactivity analysis and TUNEL assay in A375 tumor models. (a) Top panel: proliferated tumor cells were detected by an antibody to PCNA. The SKLB-163-treated group showed a significant decrease compared with the control group in a dose-dependent way. Bottom panel: detection of apoptotic cells using TUNEL analysis. The data revealed that SKLB-163 induced a significant enhancement of apoptotic cells versus control. (b) Quantified values shown were the average percentage of PCNA-positive nuclei. (c) Percent apoptosis in each group

block SKLB-163-mediated tumor suppression in vitro and in vivo. Moreover, SKLB-163 activated caspase-3 and reduced phosphorylated Akt and p44/42 MAPK, which have central roles in cell apoptosis and proliferation. In conclusion, SKLB-163 inhibited upstream RhoGDI protein and activated JNK-1 signaling pathway that could contribute to activation of caspase-3, decreased level of phosphorylated MAPK and $\mathrm{AKT}$.

RhoGDI, a cellular regulatory protein, works mainly by controlling the cellular distribution and activity of Rho GTPases. ${ }^{14,15,16}$ It belongs to a member of a family of GDIs that include D4-GDI, RhoGDI-3 and RhoGDI. RhoGDI can negatively regulate Rho proteins in three ways: (a) by shielding the membrane-anchoring domain of the GTPases, thereby restricting them to a cytosolic localization; (b) by suspending their interaction with guanine nucleotide exchange factors (GEF), thereby inhibiting GTPase activation; and (c) by blocking binding to downstream target molecules. ${ }^{17}$ RhoGDI is overexpressed in multiple types of human cancers, including ovarian cancer, breast cancer, melanoma, lung cancer and so on. ${ }^{18,19}$ Overexpression of RhoGDI is associated with tumor progression and poor prognosis of colorectal cancer. ${ }^{20}$ These findings mentioned above may help to understand RhoGDI can become a new target for anticancer treatment. However, few of specific RhoGDI inhibitors were designed and investigated.

Small-molecule inhibitors SKLB-163 have the following advantages: first, synthetic route is easy to handle and cost is low. Second, oral administration is usually safe and convenient, which patients can easily accept and adopt. Third, SKLB-163 can achieve the main goals of anticancer drug development: efficacy and safety. Finally, SKLB-163 has broad-spectrum antitumor activity in that RhoGDI is overexpressed in multiple types of human cancers. All the evidence indicates that SKLB-163 is an ideal small-molecule anticancer compound and conducive to the successful development of new drugs.

Taken together, we demonstrated that SKLB-163 had good anticancer activities in vitro and in vivo. The molecular mechanism is involved as follows: SKLB-163 inhibited upstream RhoGDI protein and activated JNK-1 signaling pathway that could contribute to activation of caspase-3, decreased level of phosphorylated MAPK and AKT. These findings will contribute to development of novel benzothiazole-2-thiol anticancer drugs.

\section{Materials and Methods}

Cell culture and synthesis of SKLB-163. Human melanoma cell line A375, human lung adenocarcinoma cell line SPC-A1, human colorectal cancer cell line SW620, human cervical cancer cell line Hela, and human prostate cancer cell line PC-3 were obtained from the American Type Culture Collection (ATCC, Manassas, VA, USA). These cells were grown in RPMI 1640 (Life Technologies, Bedford, MA, USA) or DMEM (Life Technologies) containing $10 \%$ heat-inactivated fetal bovine serum, $100 \mathrm{units} / \mathrm{ml}$ penicillin, and $100 \mathrm{units} / \mathrm{ml}$ streptomycin in a humid chamber at $37^{\circ} \mathrm{C}$ under $5 \% \mathrm{CO}_{2}$ in atmosphere.

The route adapted for the synthesis of compound SKLB-163 was done as previously described. ${ }^{10}$ It was dissolved in DMSO as a stock solution, and then was 

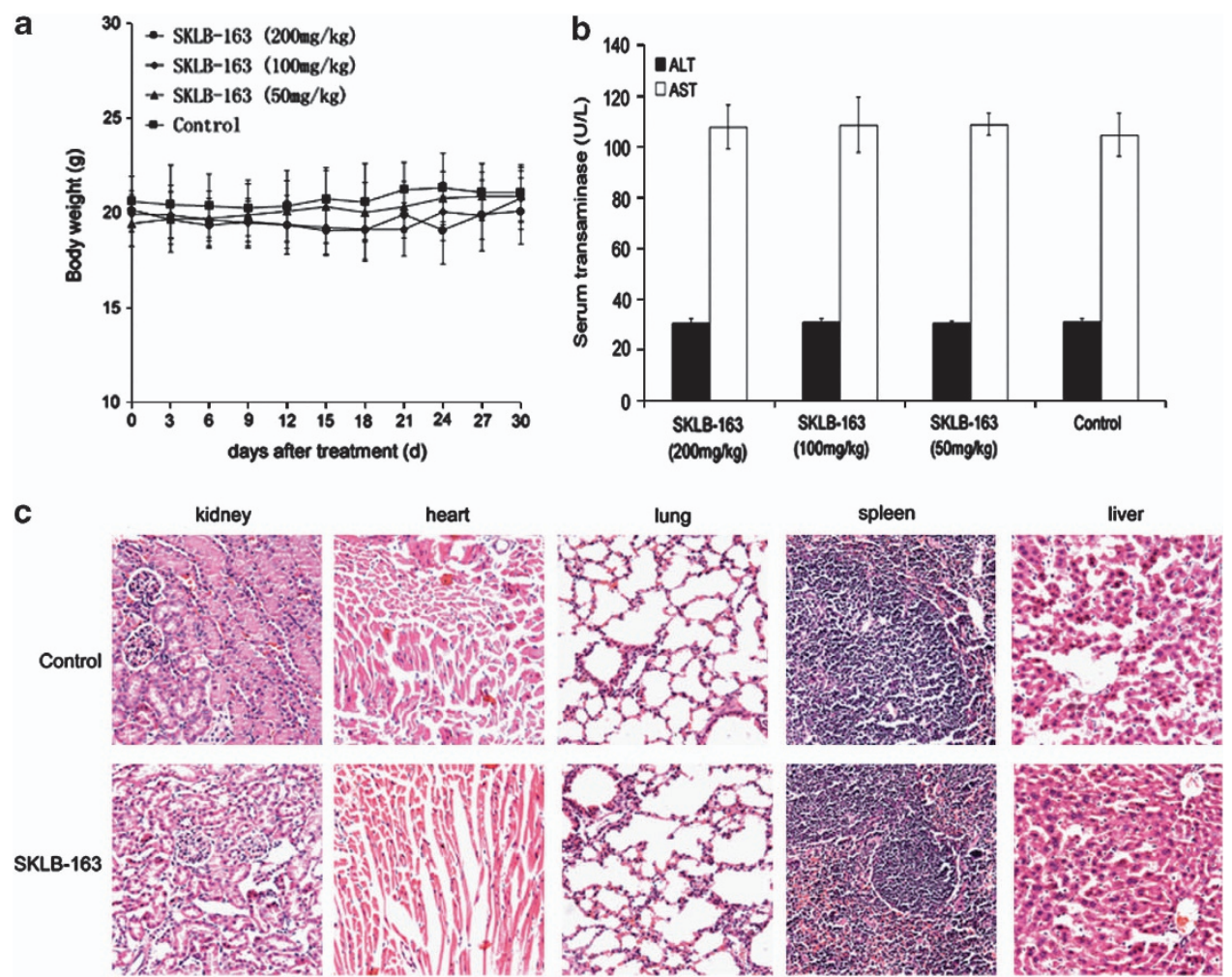

Figure 5 Analysis of systemic toxicity and potential side effects for treatment of SKLB-163. (a) Lack of toxicity-dependent body weight loss in tumor-bearing mice treated with SKLB-163. Body weights were plotted at 3-day intervals. There were no significant differences in weights among the four groups $(P>0.05)$. Values were shown as mean \pm S.D. (b) Serum ALT and AST levels of tumor-bearing mice treated with SKLB-163. No significant elevation was observed in the SKLB-163-treated groups compared with the control ( $P>0.05$, respectively). Values were shown as mean \pm S.D. (c) Representative H\&E staining of kidney, heart, lung, spleen and liver from tumor-bearing mice treated with $200 \mathrm{mg} / \mathrm{kg}$ SKLB-163 and the control group. No toxic pathological changes were seen between the two groups

Table 2 The blood toxicity of SKLB-163 treatment

\begin{tabular}{lcccc}
\hline Treatment & WBC $(\times \mathbf{1 0}$ /l) & PLT $(\times \mathbf{1 0}$ /l) & RBC $(\times \mathbf{1 0} \mathbf{1 2} / \mathbf{l})$ & HGB $(\mathbf{g} / \mathbf{l})$ \\
\hline SKLB-163(200 $\mathrm{mg} / \mathrm{kg})$ & $7.50 \pm 0.15$ & $568.8 \pm 12.9$ & $8.37 \pm 0.05$ & $138.9 \pm 2.84$ \\
SKLB-163(100 $\mathrm{mg} / \mathrm{kg})$ & $7.50 \pm 1.03$ & $565.6 \pm 12.1$ & $8.54 \pm 0.06$ & $140.4 \pm 4.76$ \\
SKLB-163(50 mg/kg) & $7.56 \pm 0.32$ & $563.4 \pm 7.3$ & $8.38 \pm 0.15$ & $138.7 \pm 15.39$ \\
Control & $7.54 \pm 0.16$ & $574.1 \pm 16.9$ & $8.43 \pm 0.09$ & $147.3 \pm 17.80$ \\
\hline
\end{tabular}

Data are expressed as the mean \pm S.D.

stored at $4{ }^{\circ} \mathrm{C}$. In in vitro study, the stock solution was diluted in cell culture medium at final DMSO concentration of $0.05 \%(\mathrm{v} / \mathrm{v})$. The formulation used in in vivo study contains SKLB-163, carboxymethylcellulose $0.5 \%$ and glycerin $1 \%$.

Cell viability assay. The cell viability of SKLB-163-treated cancer cells was determined by the MTT assay. Briefly, cells $\left(4-5 \times 10^{3}\right)$ were seeded in 96-well plates and cultured for $24 \mathrm{~h}$, followed by SKLB-163 treatment for $48 \mathrm{~h}$. A volume of $10 \mu \mathrm{l} \mathrm{of} 10 \mathrm{mg} / \mathrm{ml} \mathrm{MTT}$ was added per well and incubated for another $3 \mathrm{~h}$ at $37^{\circ} \mathrm{C}$, then the supernatant fluid was removed and DMSO was added at $150 \mu \mathrm{l} /$ well for 15-20 min. The light absorptions (OD) were measured at $570 \mathrm{~nm}$ with SpectraMAX M5 microplate spectrophotometer (Molecular Devices, Sunnyvale, CA, USA). The effect of SKLB-163 on tumor cells viability was expressed by $I_{50}$ of each cell lines.

Morphological analysis. A375 cells were seeded in six-well plates at the density of $3 \times 10^{5} /$ well and cultured for $24 \mathrm{~h}$, followed by SKLB-163 treatment for $48 \mathrm{~h}$, and then collected and washed with PBS. The cells were fixed using $70 \%$ of ethanol following rinsing with PBS. Then the cells were stained with Hoechst $(5 \mu \mathrm{g} / \mathrm{ml})$ and analyzed under fluorescence microscope (Zeiss, Axiovert 200, Oberkochen, Germany) to identify the apoptotic cells.
The quantitative assessment of apoptosis. Apoptosis cells treated with SKLB-163 was further analyzed by a flow cytometer. Collected cells were stained with $1 \mathrm{ml}$ hypotonic fluorochrome solution containing $50 \mu \mathrm{g} / \mathrm{ml}$ propidium iodide in $0.1 \%$ sodium citrate plus $0.1 \%$ Triton $X-100$. Then flow cytometric analysis could be performed to identify apoptotic cells or sub-G1 cells and to measure the percentage of sub-G1 cells by the use of a flow cytometer (ESP Elite; Coulter, Brea, CA, USA). Apoptotic cells has a less DNA content than that of G1 cells in the cell cycle distribution and the results were estimated with list mode software.

Clonogenic survival determination. A375 cells were assayed for colony-forming ability by replating them in specified numbers (300-400/well) in six-well plates and treated with $0.625,1.25$, and $2.5 \mu \mathrm{M}$ of SKLB-163, respectively. After 14 days of incubation, the cells were stained with $0.5 \%$ crystal violet in absolute ethanol and colonies were counted under dissection microscope. ${ }^{21}$

In vivo tumor experiment. To study the antitumor activities of SKLB-163 in vivo, A375 human melanoma and SPC-A1 human lung adenocarcinoma models were established. In brief, $4 \times 10^{6}$ A375 or $5 \times 10^{6}$ SPC-A1 cells were subcutaneously injected into the right dorsal flank of 6 - to 8-week-old female 
a
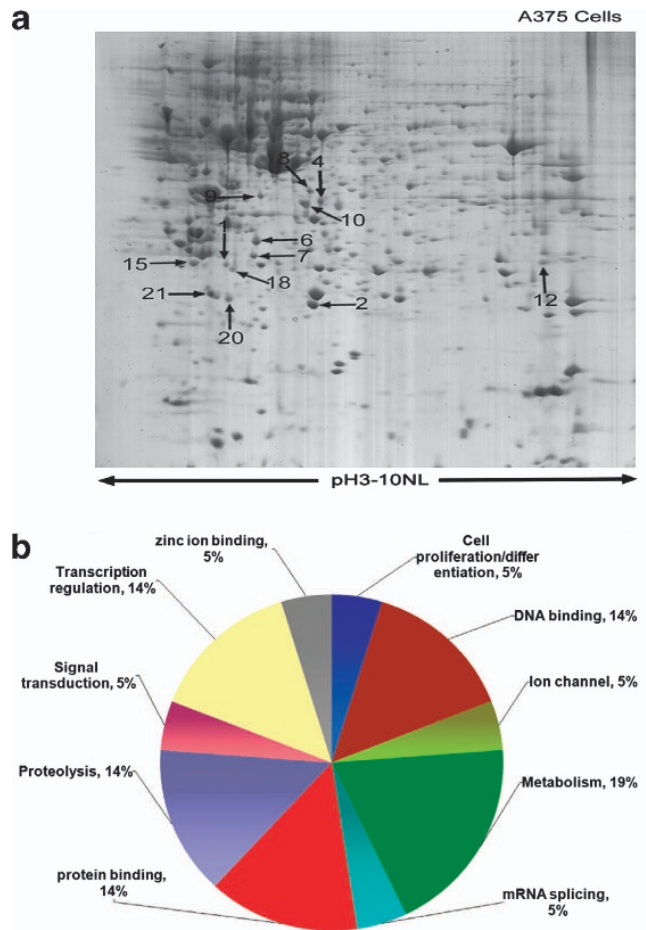

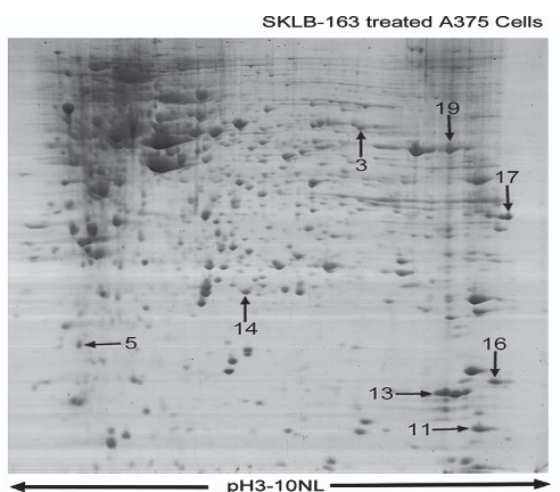

C

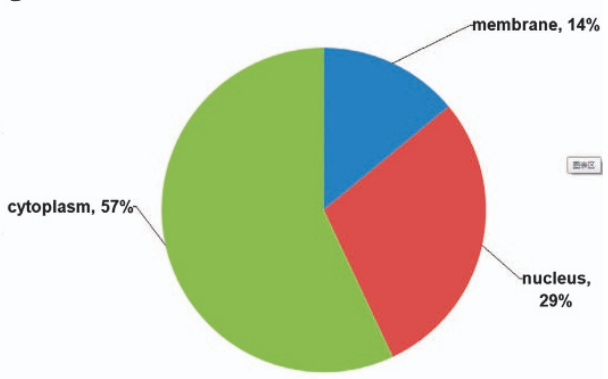

Figure 6 2-DE profiling of the differentially expressed proteins between A375 and SKLB-163-treated A375 cells. (a) Protein samples (2 mg) from A375 cells or SKLB-163 (2.5 $\mu \mathrm{M} ; 48 \mathrm{~h})$-treated A375 cells were separated on 2-DE gel and visualized by Coomassie blue staining. Arrows indicated identified protein spots significantly and consistently altered between A375 and SKLB-163-treated A375 cells. (b) Cluster analysis revealed that the altered proteins were involved in diverse biological processes, including metabolism (19\%), transcription regulation (14.3\%), proteolysis (14.3\%) and so on. (c) The identified proteins were categorized into groups according to their subcellular locations. Among them, $57 \%$ of the proteins were located in the cytoplasm and others were located in the nucleus (29\%) or membrane (14\%) as indicated

Table 3 Identified proteins by MS/MS analysis

\begin{tabular}{|c|c|c|c|c|c|c|c|c|}
\hline $\begin{array}{l}\text { Spot } \\
\text { no. }\end{array}$ & Protein description & Gene name & Function & $\begin{array}{c}\text { Accession } \\
\text { no. }\end{array}$ & $\mathrm{Mr}^{\mathrm{a}}$ & Score & $\begin{array}{l}\text { No. of } \\
\text { pep }^{b}\end{array}$ & $\begin{array}{c}\text { Fold } \\
\text { change }^{c}\end{array}$ \\
\hline 1 & Calpain small subunit 1 & CAPNS1 & Cell proliferation/differentiation & P04632 & 28469 & 432 & 41 & $\downarrow 2.3 \pm 0.6$ \\
\hline 2 & Rho GDP-dissociation inhibitor 1 & ARHGDIA & Metabolism & P52565 & 23250 & 135 & 12 & $\downarrow 3.6 \pm 0.5$ \\
\hline 3 & D-3-phosphoglycerate dehydrogenase & PHGDH & Metabolism & 043175 & 57356 & 94 & 17 & $\uparrow 2.1 \pm 0.3$ \\
\hline 4 & $60 \mathrm{~S}$ acidic ribosomal protein PO & RPLPO & Protein binding & P05388 & 34423 & 251 & 19 & $2.4 \pm 0.5$ \\
\hline 5 & S-phase kinase-associated protein 1 & SKP1 & Proteolysis & $\mathrm{P} 63208$ & 18817 & 205 & 11 & $\uparrow 2.2 \pm 0.2$ \\
\hline 6 & Chloride intracellular channel protein 1 & CLIC1 & Ion channel & 000299 & 27248 & 215 & 12 & $4.3 \pm 1.4$ \\
\hline 7 & Proteasome subunit alpha type- 3 & PSMA3 & Proteolysis & P25788 & 28643 & 25 & 5 & $2.0 \pm 0.3$ \\
\hline 8 & $\begin{array}{l}\text { Pyruvate dehydrogenase E1 component subunit } \\
\text { beta, mitochondrial }\end{array}$ & PDHB & Metabolism & P11177 & 39550 & 231 & 13 & $\downarrow 2.3 \pm 0.4$ \\
\hline 9 & Protein SEC13 homolog & SEC13 & Protein binding & P55735 & 36031 & 42 & 1 & $\downarrow 3.2 \pm 0.8$ \\
\hline 10 & Eukaryotic translation initiation factor 3 subunit I & EIF3I & Transcription regulation & Q13347 & 36878 & 86 & 15 & $\downarrow 5.6 \pm 1.3$ \\
\hline 11 & Single-stranded DNA-binding protein, mitochondrial & SSBP1 & DNA binding & Q04837 & 17249 & 109 & 9 & $\uparrow 2.0 \pm 0.2$ \\
\hline 12 & Eukaryotic translation initiation factor $4 \mathrm{H}$ & EIF4H & Transcription regulation & Q15056 & 27425 & 70 & 3 & $\downarrow N A$ \\
\hline 13 & Histone $\mathrm{H} 2 \mathrm{~B}$ type $1-\mathrm{B}$ & HIST1H2BB & DNA binding & P33778 & 13942 & 288 & 11 & $\uparrow \mathrm{NA}$ \\
\hline 14 & Protein DJ-1 & PARK7 & Protein binding & Q99497 & 20050 & 166 & 10 & $\uparrow 3.7 \pm 1.3$ \\
\hline 15 & Proteasome subunit alpha type- 5 & PSMA5 & Proteolysis & P28066 & 26565 & 103 & 11 & $\downarrow 3.3 \pm 0.7$ \\
\hline 16 & Nucleoside diphosphate kinase B & NME2 & DNA binding & P22392 & 17401 & 124 & 29 & $\uparrow 3.1 \pm 1.2$ \\
\hline 17 & Heterogeneous nuclear ribonucleoproteins A2/B1 & HNRNPA2B1 & mRNA splicing & P22626 & 37464 & 197 & 17 & $\uparrow 2.2 \pm 0.6$ \\
\hline 18 & Histone $\mathrm{H} 2 \mathrm{~A}$ type 2-A & $\mathrm{H} 2 \mathrm{~A} 2 \mathrm{~A}$ & Transcription regulation & Q6FI13 & 14087 & 153 & 6 & $2.1 \pm 0.2$ \\
\hline 19 & Retinal dehydrogenase 1 & ALDH1A1 & Metabolism & P01834 & 55454 & 196 & 20 & $\uparrow 3.6 \pm 1.6$ \\
\hline 20 & Lactoylglutathione lyase & GLO1 & Zinc ion binding & Q04760 & 20992 & 65 & 12 & $\downarrow 2.6 \pm 0.9$ \\
\hline 21 & Ras-related protein Rab-7a & RAB7A & Signal transduction & P51149 & 23760 & 46 & 2 & $\downarrow 2.0 \pm 0.3$ \\
\hline
\end{tabular}

All protein spots identified by ESI-Q-TOF MS/MS as significantly changed in average expression level in SKLB-163-treated A375 cells compared with the parental A375 cells

${ }^{\mathrm{a}} \mathrm{Mr}$ : Theoretical molecular weight $(\mathrm{kDa})$ from the ExPASy database

${ }^{b}$ No. of pep: number of unique peptides identified by MS/MS sequencing

'Upward arrows, upregulated; downward arrows, downregulated; NA, the spots on one of the paired gels were too weak or nondetectable

athymic nude BALB/C mice. When the tumor diameters were approximately $0.6-0.8 \mathrm{~cm}$, the tumor-bearing mice were randomly assigned into the following four groups (ten mice per treatment group) and each mouse received the corresponding treatment by intragastric administration once daily: (a) control group; (b) SKLB-163, 50 mg/kg; (c) SKLB-163, 100 mg/kg; (d) SKLB-163, $200 \mathrm{mg} / \mathrm{kg}$. Tumor volumes were evaluated according to the following formula: tumor volume 
a

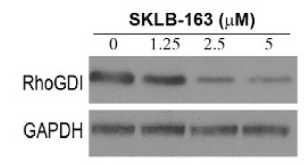

d
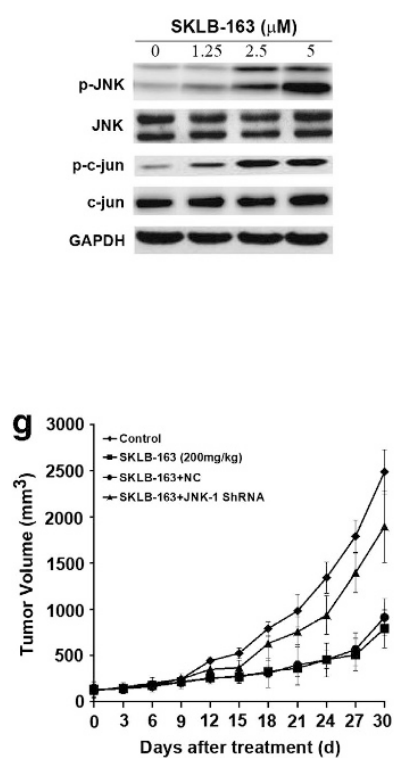

b

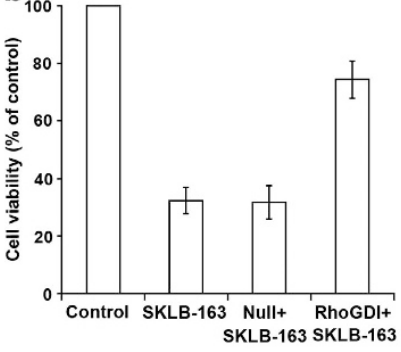

e

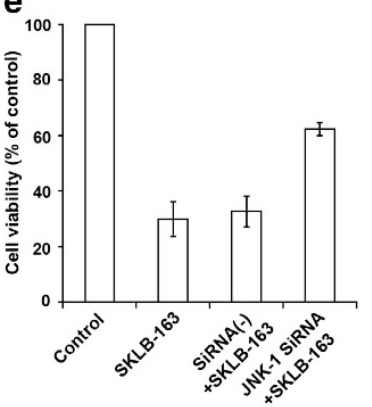

h

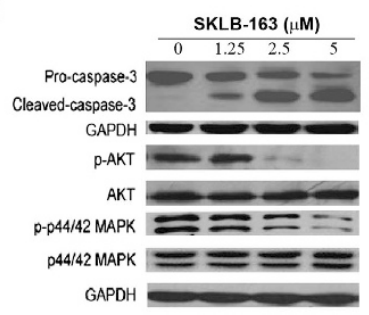

C

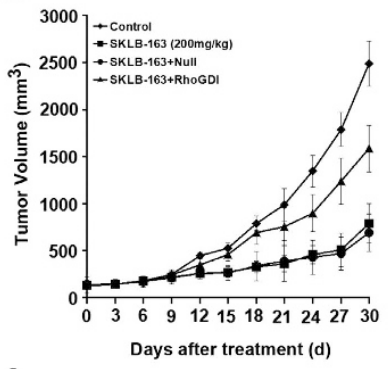

f

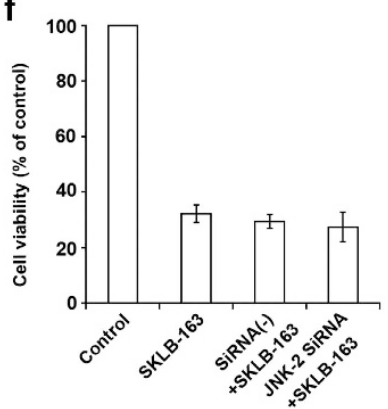

i

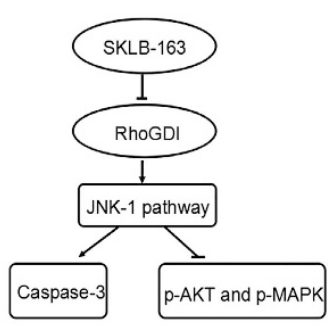

Figure 7 (a) Validated downregulation of RhoGDI in SKLB-163-treated A375 cells compared with the parental A375 cells by western blot analysis using RhoGDI-specific antibody. (b) The effect of RhoGDI overexpression on cytotoxicity of SKLB-163. (c) Overexpression of RhoGDI markedly rescued tumor cells from SKLB-163-induced tumor suppression in vivo. (d) SKLB-163 dose-dependently activated JNK and its downstream target c-jun in A375 cells. (e) The effect of JNK-1 silencing on cytotoxicity of SKLB163. (f) The effect of JNK-2 silencing on cytotoxicity of SKLB-163. (g) Silencing JNK-1 could block SKLB-163-mediated tumor suppression in vivo. (h) SKLB-163 increased cleaved caspase-3 and inhibited Akt and p 44/42 MAPK phosphorylation. A375 cells were treated with SKLB-163 at various concentrations (0-5 $\mu \mathrm{M})$ for $48 \mathrm{~h}$ and then we analyzed the expression levels of pro-caspase-3, cleaved caspase-3, Akt, p 44/42 MAPK and their phosphorylation status by western blotting. (i) A possible schematic model to better illustrate the action mechanism of SKLB-163

$\left(\mathrm{mm}^{3}\right)=0.52 \times$ length $\times$ width $^{2}$. The side effects of treatment and the weight, appetite, and behavior of the mice were recorded for 30 days, after which the mice were killed.

Tumor net weight of each mouse was measured. Organs (lung, liver, kidney, heart and spleen) and tumors from killed mice were removed, fixed in the $4 \%$ formaldehyde solution for histological analysis.

Immunohistochemistry. The tumor sections were stained by the Envision System-HRP method (DakoCytomation Inc, Carpinteria, CA, USA), according to the kit manufacturer's instructions. The primary antibodies for PCNA were purchased from Santa Cruz Biotechnology (Santa Cruz, CA, USA).

TUNEL assay. The presence of apoptotic cells within the tumor sections was evaluated by the TUNEL (terminal deoxynucleotidyltransferase-mediated dUTP nick-end labeling) technique using the DeadEnd Fluorometric TUNEL System (Promega, Madison, WI, USA) following the manufacturer's protocol. Percent apoptosis was determined by counting the number of apoptotic cells and dividing by the total number of cells in the field ( 5 high power fields/slide).

Toxicity assessment. To evaluate the potential side effects in the SKLB163-treated mice, they were continuously observed for relevant indexes such as weight loss, ruffled fur, diarrhea, anorexia, skin ulcer or toxic deaths. After killing, various organs (lung, liver, kidney, heart, and spleen) were collected and fixed in
$4 \%$ formaldehyde solution. These tissues were then sectioned, stained with H\&E, and observed by two pathologists in a blinded manner.

Besides, to evaluate the liver toxicity, levels of serum ALT and AST were analyzed. The levels of serum ALT and AST were determined with an automatic multifunction-biochemical analyzer. In addition, to assess the potential blood toxicity induced by SKLB-163 treatment, peripheral blood of the mice was analyzed. Complete blood counts and differentials were measured within each sample using an Abbott CELL-DYN 3700 hematology analyzer (Abbott Laboratories, Abbott Park, IL, USA). Peripheral blood counts were depicted as average values \pm S.D.

2-DE and image analysis. The 2-DE was performed as described previously with minor modification. ${ }^{22}$ Approximately $4 \times 10^{7}$ cells were lysed in $1 \mathrm{ml}$ lysis buffer (7M urea, $2 \mathrm{M}$ thiourea, $4 \%$ CHAPS, $100 \mathrm{mM}$ DTT, $0.2 \%$ (pH 3-10) ampholyte, Bio-Rad, Berkeley, CA, USA) containing protease inhibitor cocktail 8340 (Sigma, St. Louis, MO, USA). Samples were then kept on ice and sonicated in six cycles of $15 \mathrm{~s}$, each consisting of $5 \mathrm{~s}$ sonication followed by a 10-s break. After centrifugation at 15000 r.p.m. for $1 \mathrm{~h}$ at $4{ }^{\circ} \mathrm{C}$, the supernatant was collected and the protein concentrations were determined using the DC protein assay kit (Bio-Rad). Protein samples $(2 \mathrm{mg})$ were applied to IPG strip $(17 \mathrm{~cm}, \mathrm{pH}$ 3-10 NL, Bio-Rad) using a passive rehydration method. After $12-16 \mathrm{~h}$ of rehydration, the strips were transferred to an IEF Cell (Bio-Rad). IEF was performed as follows: $250 \mathrm{~V}$ for $30 \mathrm{~min}$, linear; $1000 \mathrm{~V}$ for $1 \mathrm{~h}$, rapid; linear ramping to $10000 \mathrm{~V}$ for $5 \mathrm{~h}$ and finally $10000 \mathrm{~V}$ for $4 \mathrm{~h}$. The second dimension was 
performed using $12 \%$ SDS-PAGE at $30 \mathrm{~mA}$ constant current per gel after equilibration. The gels were stained using CBB R-250 (Merck, Darmstadt, Germany) and scanned with a Bio-Rad GS-800 scanner. Three independent runs were made for each cell lines to ensure the accuracy of analyses. The maps were analyzed by PDQuest software Version 6.1 (Bio-Rad). The quantity of each spot in a gel was normalized as a percentage of the total quantity of all spots in that gel and evaluated in terms of OD. For statistical analysis, paired t-test was performed to compare data from the three repeated experiments. Only spots that showed consistent and significant differences ( \pm over twofold, $P<0.05$ ) were selected for analysis with MS.

In-gel digestion. In-gel digestion of proteins was carried out using MS-grade Trypsin Gold (Promega) according to the manufacturer's instructions. Briefly, spots were cut out of the gel (1-2 mm diameter) using a razor blade, and destained twice with $100 \mathrm{mM} \mathrm{NH}{ }_{4} \mathrm{HCO}_{3} / 50 \% \mathrm{ACN}$ at $37^{\circ} \mathrm{C}$ for $45 \mathrm{~min}$ in each treatment. After drying, the gels were preincubated in 10-20 $\mu \mathrm{l}$ trypsin solution for $1 \mathrm{~h}$. Then, $15 \mu$ l digestion buffer was added $\left(40 \mathrm{mM} \mathrm{NH}_{4} \mathrm{HCO}_{3} / 10 \% \mathrm{ACN}\right)$ to cover each gel and incubated overnight at $37^{\circ} \mathrm{C}$. Tryptic digests were extracted using MilliQ water initially, followed by two times extraction with $50 \% \mathrm{ACN} / 5 \%$ TFA for $1 \mathrm{~h}$ each time. The combined extracts were dried in a vacuum concentrator at room temperature. The samples were then subjected to MS analysis.

ESI-Q-TOF analysis and protein identification. Mass spectra were acquired using a Q-TOF mass spectrometer (Micromass, Manchester, UK) fitted with an ESI source (Waters, Manchester, UK). Tryptic digests were dissolved in $18 \mu \mathrm{l} 50 \% \mathrm{ACN}$. MS/MS was performed in a data-dependent mode in which the top ten most abundant ions for each MS scan were selected for MS/MS analysis. Trypsin autolysis products and keratin-derived precursor ions were automatically excluded. The MS/MS data were acquired and processed using MassLynx software (Micromass) and MASCOT (http://www.matrixscience.com) was used to search the database. Database searches were carried out using the following parameters: Database, Swiss-Prot; taxonomy, Homo sapiens; enzyme, trypsin; mass tolerance, $\pm 0.1 \mathrm{Da}$; MS/MS tolerance, $\pm 0.05 \mathrm{Da}$; and an allowance of one missed cleavage. Fixed modifications of cysteine carboamidomethylation, and variable modifications of methionine oxidation were allowed. The data format was selected as Micromass PKL and the instrument was selected as ESI-Q-TOF. Proteins with probability-based MOWSE scores, derived from ions scores as a nonprobabilistic basis for ranking protein hits when using Mascot searching engine, exceeding their threshold $(P<0.05)$ were considered to be positively identified. To eliminate the redundancy of proteins appearing in the database under different names or accession numbers, the one-protein member with the highest MASCOT score was further selected from the relevant multiple-member protein family.

Western blotting. Proteins were extracted in RIPA buffer $(50 \mathrm{mM}$ Tris-base, $1.0 \mathrm{mM}$ EDTA, $150 \mathrm{mM} \mathrm{NaCl}, 0.1 \%$ SDS, $1 \%$ Triton X-100, $1 \%$ sodium deoxycholate, $1 \mathrm{mM}$ PMSF) and quantified by the DC protein assay kit (Bio-Rad). Samples were separated by $12 \%$ SDS-PAGE and transferred to PVDF membranes (Amersham Biosciences, Piscataway, NJ, USA). After blocking with $5 \%$ non-fat milk in Tris-buffered saline, $0.1 \%$ Tween 20 for $1 \mathrm{~h}$, the membranes was incubated overnight at $4{ }^{\circ} \mathrm{C}$ with respective primary antibodies. The primary antibodies for RhoGDI, JNK, p-JNK, Akt/p-Akt and MAPK/p-MAPK were purchased from Cell Signaling Technology (Beverly, MA, USA). The primary antibodies for c-Jun, p-c-Jun, caspase-3 and GAPDH were acquired from Santa Cruz Biotechnology. After that, the blots were incubated with secondary antibody conjugated to HRP for $2 \mathrm{~h}$ at room temperature. Target proteins were detected by enhanced chemiluminescence reagents (Amersham Pharmacia Biotech, Piscataway, NJ, USA).

RhoGDI overexpression. RhoGDI plasmids were synthesized by Western Biotechnology Inc (Chongqing, China). Cancer cells were transfected with $2 \mu \mathrm{g}$ plasmids plus $5 \mu \mathrm{g}$ Lipofectamine 2000 reagent (Invitrogen, Carlsbad, CA, USA). Cell viability experience was conducted as described above.

As described above, A375 human melanoma models were established. When the tumor diameters were about $0.6-0.8 \mathrm{~cm}$, the tumor-bearing mice were randomly assigned into the following four groups: (a) control group; (b) SKLB-163, $200 \mathrm{mg} / \mathrm{kg}$; (c) SKLB-163, $200 \mathrm{mg} / \mathrm{kg}$ + negative control, $50 \mu \mathrm{g}+$ Lipofectamine 2000/125 $\mu \mathrm{g}$; (d) SKLB-163, $200 \mathrm{mg} / \mathrm{kg}$ + RhoGDI plasmids, $50 \mu \mathrm{g}+$ Lipofectamine 2000/125 $\mu \mathrm{g}$. Tumor volumes were evaluated as described above.

JNK-1 and JNK-2 RNAi. JNK-1 siRNA and JNK-2 siRNA were synthesized by RiboBio (Guangzhou, China). The siRNA sequences against JNK-1 or JNK-2 were previously described. Cells were transfected with $1 \mu \mathrm{g}$ siRNA using $2.5 \mu \mathrm{g}$ Lipofectamine 2000 reagent (Invitrogen). Scrambled siRNA, which consists of a scrambled sequence that will not lead to specific degradation of any known cellular mRNA, was employed as negative control. Cell viability experience was conducted as described above.

JNK-1 shRNA were also synthesized by RiboBio (Guangzhou, China). As described above, A375 human melanoma models were established. When the tumor diameters were about $0.6-0.8 \mathrm{~cm}$, the tumor-bearing mice were randomly assigned into the following four groups: (a) control group; (b) SKLB-163, $200 \mathrm{mg} / \mathrm{kg}$; (c) SKLB-163, $200 \mathrm{mg} / \mathrm{kg}$ + negative control, $50 \mu \mathrm{g}+$ Lipofectamine 2000/125 $\mu \mathrm{g}$; (d) SKLB-163, $200 \mathrm{mg} / \mathrm{kg}+$ JNK-1 ShRNA, $50 \mu \mathrm{g}+$ Lipofectamine 2000/125 $\mu \mathrm{g}$. Tumor volumes were evaluated as described above.

Statistical analysis. Statistical analysis of the differences in tumor volume, tumor net weight, animal weight, percent apoptosis, percentages of PCNA-positive nuclei and the level of serum transaminase were performed using one-way analysis of variance. $P<0.05$ was considered statistically significant.

\section{Conflict of Interest}

The authors declare no conflict of interest.

1. Varmus $H$. The new era in cancer research. Science 2006; 312: 1162-1165

2. Hawk E, Viner JL. What is the future of oncology? National Cancer Institute initiatives to improve research, development, and implementation in cancer prevention and treatment. Semin Oncol 2006; 33(6 Suppl 11): S6-S9.

3. Berardi R, Santoni M, Morgese F, Ballatore Z, Savini A, Onofri A et al. Novel small molecule EGFR inhibitors as candidate drugs in non-small cell lung cancer. OncoTargets Therapy 2013; 6: 563-576

4. Druker BJ. Imatinib as a paradigm of targeted therapies. Adv Cancer Res 2004; 91: 1-30.

5. Hutchinson I, Chua MS, Browne HL, Trapani V, Bradshaw TD, Westwell AD et al. Antitumor benzothiazoles. 14. Synthesis and in vitro biological properties of fluorinated 2-(4-aminophenyl)benzothiazoles. J Med Chem 2001; 44: 1446-1455.

6. Kok SH, Gambari R, Chui CH, Yuen MC, Lin E, Wong RS et al. Synthesis and anti-cancer activity of benzothiazole containing phthalimide on human carcinoma cell lines. Bioorg Med Chem 2008; 16: 3626-3631.

7. Trapani G, Franco M, Latrofa A, Reho A, Liso G. Synthesis, in vitro and in vivo cytotoxicity, and prediction of the intestinal absorption of substituted 2-ethoxycarbonyl-imidazo[2,1-b] benzothiazoles. Eur J Pharmaceut Sci 2001; 14: 209-216.

8. Song EY, Kaur N, Park MY, Jin Y, Lee K, Kim G et al. Synthesis of amide and urea derivatives of benzothiazole as Raf-1 inhibitor. Eur J Med Chem 2008; 43: 1519-1524.

9. Srimanth K, Rao VR, Krishna DR. Synthesis and evaluation of anticancer activity of some imidazothiazolyl, imidazobenzothiazolyl and dihydroimidazothiazolyl coumarins. Arzneimittel-Forschung 2002; 52: 388-392.

10. Wang Z, Shi XH, Wang J, Zhou T, Xu YZ, Huang TT et al. Synthesis, structure-activity relationships and preliminary antitumor evaluation of benzothiazole-2-thiol derivatives as novel apoptosis inducers. Bioorg Med Chem Lett 2011; 21: 1097-1101.

11. Park YJ, Ahn HJ, Chang HK, Kim JY, Huh KH, Kim MS et al. The RhoGDI-alpha/JNK signaling pathway plays a significant role in mycophenolic acid-induced apoptosis in an insulin-secreting cell line. Cell Signal 2009; 21: 356-364.

12. Shigetomi H, Higashiura $Y$, Kajihara H, Kobayashi $H$. Targeted molecular therapies for ovarian cancer: an update and future perspectives (Review). Oncol Rep 2012; 28: 395-408.

13. Novello S, Giaj Levra M, Vavala T. Functional imaging in predicting response to antineoplastic agents and molecular targeted therapies in lung cancer: a review of existing evidence. Crit Rev Oncol Hematol 2012; 83: 208-215.

14. Olofsson B. Rho guanine dissociation inhibitors: pivotal molecules in cellular signalling. Cell Signal 1999; 11: 545-554

15. Sasaki T, Takai Y. The Rho small G protein family-Rho GDI system as a temporal and spatial determinant for cytoskeletal control. Biochem Biophys Res Commun 1998; 245 : 641-645.

16. Golovanov AP, Chuang TH, DerMardirossian C, Barsukov I, Hawkins D, Badii R et al. Structure-activity relationships in flexible protein domains: regulation of rho GTPases by RhoGDI and D4 GDI. J Mol Biol 2001; 305: 121-135.

17. Zhang B, Zhang Y, Dagher MC, Shacter E. Rho GDP dissociation inhibitor protects cancer cells against drug-induced apoptosis. Cancer Res 2005; 65: 6054-6062.

18. Poland J, Schadendorf D, Lage H, Schnolzer M, Celis JE, Sinha P. Study of therapy resistance in cancer cells with functional proteome analysis. Clin Chem Lab Med 2002; 40: 221-234.

19. Sinha P, Kohl S, Fischer J, Hutter G, Kern M, Kottgen E et al. Identification of novel proteins associated with the development of chemoresistance in malignant melanoma using twodimensional electrophoresis. Electrophoresis 2000; 21: 3048-3057.

20. Zhao L, Wang H, Li J, Liu Y, Ding Y. Overexpression of Rho GDP-dissociation inhibitor alpha is associated with tumor progression and poor prognosis of colorectal cancer. J Proteome Res 2008; 7: 3994-4003. 
21. Wang X, Wei Y, Yuan S, Liu G, Zhang YL, Wang W. Potential anticancer activity of litchi fruit pericarp extract against hepatocellular carcinoma in vitro and in vivo. Cancer Lett 2006; 239: 144-150.

22. Peng XC, Gong FM, Zhao YW, Zhou LX, Xie YW, Liao HL et al. Comparative proteomic approach identifies PKM2 and cofilin-1 as potential diagnostic, prognostic and therapeutic targets for pulmonary adenocarcinoma. PLoS One 2011; 6: e27309.
Cell Death and Disease is an open-access journal published by Nature Publishing Group. This work is licensed under a Creative Commons Attribution-NonCommercialNoDerivs 3.0 Unported License. To view a copy of this license, visit http://creativecommons.org/licenses/by-nc-nd/3.0/ 